\title{
Improvement at Network Planning using Heuristic Algorithm to Minimize Cost of Distance between Nodes in Wireless Mesh Networks
}

\author{
Shivan Qasim Ameen, Ravie Chandren Muniyandi \\ SOFTAM Department, Faculty of Information Science and Technology, Universiti Kebangsaan Malaysia, Malaysia
}

\begin{tabular}{l} 
Article Info \\
\hline Article history: \\
Received Oct 12, 2016 \\
Revised Dec 20, 2016 \\
Accepted Jan 4, 2017
\end{tabular}

Keyword:

Heuristic algorithm

MBPSO

Minimize cost of distance

Routers

Wireless mesh networks

\begin{abstract}
Wireless Mesh Networks (WMN) consists of wireless stations that are connected with each other in a semi-static configuration. Depending on the configuration of a WMN, different paths between nodes offer different levels of efficiency. One areas of research with regard to WMN is cost minimization. A Modified Binary Particle Swarm Optimization (MBPSO) approach was used to optimize cost. However, minimized cost does not guarantee network performance. This paper thus, modified the minimization function to take into consideration the distance between the different nodes so as to enable better performance while maintaining cost balance. The results were positive with the PDR showing an approximate increase of $17.83 \%$ whereas the E2E delay saw an approximate decrease of $8.33 \%$.
\end{abstract}

\section{Copyright $\odot 2017$ Institute of Advanced Engineering and Science.} All rights reserved.

\section{Corresponding Author:}

Shivan Qasim Ameen,

SOFTAM Departement,

Faculty of Information Science and Technology,

University Kebangsaan Malaysia (UKM),

Jalan Reko, BaruBangi, Selangor Darul Ehsan 43600, Malaysia.

Email: Shvan_8990@yahoo.com

\section{INTRODUCTION}

Wireless Mesh Network (WMN) is defined as a semi-static type of multi-hop wireless network where a number of wireless stations are connected to one or more gateways. The importance of (WMN) led to be used in several areas such as [1-12]. These wireless stations in (WMN) are known to have very limited mobility or no mobility at all [13]. By using a mesh network protocol, the stations which are commonly powered by battery communicate with each other to broadcast messages and execute other tasks. There are two types of nodes in WMN; mesh routers and mesh clients. In order to support mesh networking, mesh routers have additional routing functions such as its multiple built-no wireless interface to improve the flexibility of the network and its low transmission power through multi-hop communication. A WMN is also reliable and offers redundancy. If one node fails to operate, the rest of the nodes in network can still communicate with one another as its neighbor will simply find another route. Due to dynamic routing, nodes have the capability to choose the quickest path to send information. In a full mesh topology, every node interacts and communicates with each other not just back and forth to the central router and this helps configure routes in a more dynamic manner.

It is evident that WMNs are self-configuring and self-healing. However, there are imminent issues that these networks face. A large number of open researches discuss WMN limitations such as load balancing, energy optimization, route optimization, cost minimization, unsplittable flow, and limited resources. This work focuses on cost minimization for establishing a network by using heuristic algorithms. 
In a mesh network communication, the devices can be generally categorized into three; clients, routers and gateways. Gateways provide services for the network while routers relay the data that travels between gateways and the network subscribers. Figure 1, shows the infrastructure of a WMN.

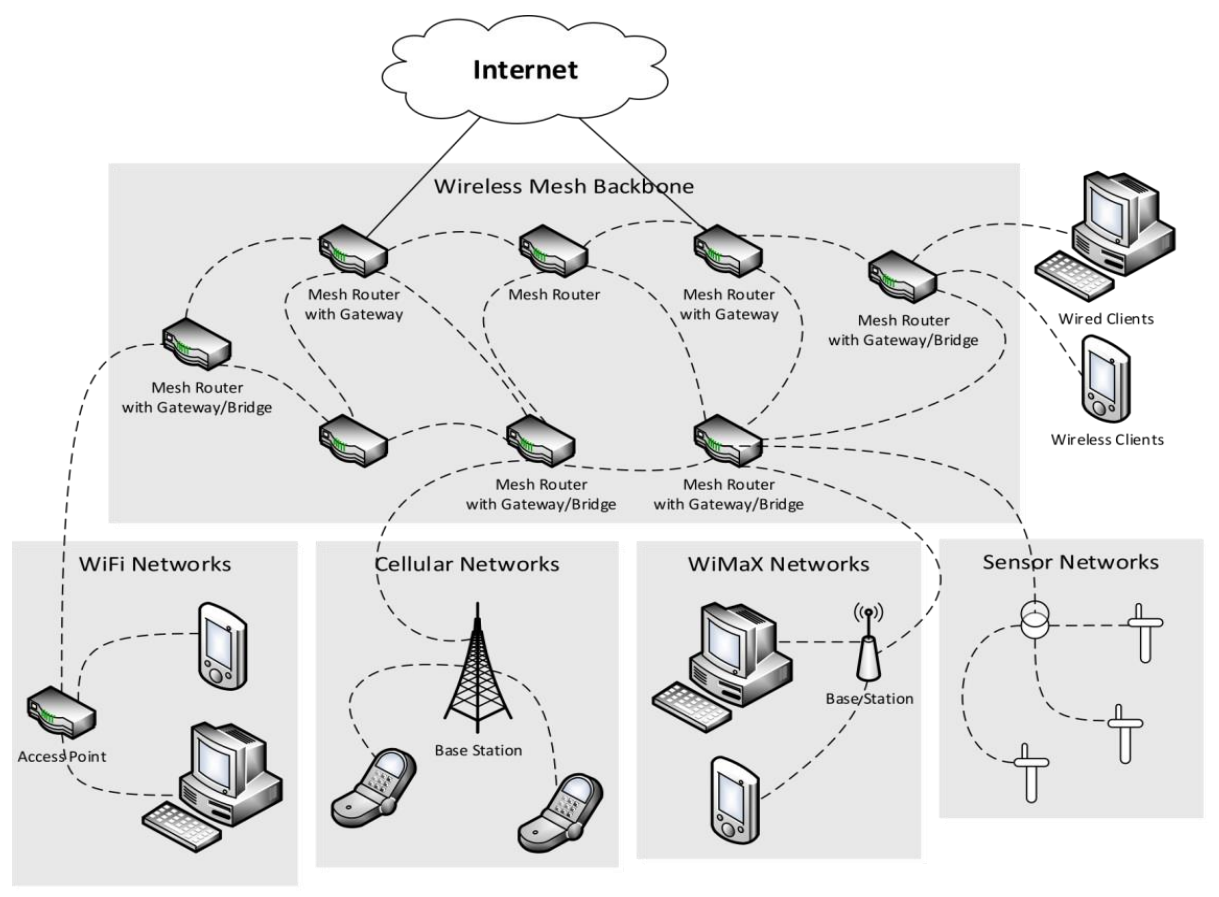

Figure 1. WMN infrastructure. Adapted from [14]

In Figure 1, the dashed and solid lines indicate wireless and wired links respectively. For medium access control, the infrastructure can be reduced to base, relay and subscriber. In this case, it is assumed that information or data packet must travel from a base station (BS) to a subscriber station (SS) via relay station (RS) in an unsplittable flow although it is possible to route the packet in a multi-path manner.

Nodes may interfere with one another when they are rebroadcasting at the same time within a small region on the same channel. This is called dense mesh network dilemma. Among the ways to resolve this issue is splitting network resources in their own channel such as splitting time in time channel and frequency in frequency channel [15]. Now that there are resources need to be handled, a question of how to allocate these available resources arises while taking other factors into consideration as well.

In wireless network route planning, resource allocation plays an important role to give a cost-andenergy-efficient solution. Heuristic approach to solve path planning in wireless network is seen as one of the most popular research areas in the recent years [16].

Proposed a heuristic algorithm to minimize the number of transmission tree and indirectly conserve bandwidth. The proposed algorithm however was built to allow multicast protocol instead of single-path. In another paper [17], implemented ant colony optimization into their anycast routing protocol in the aim to select proper access gateway in WMN. Combining distributed computing with heuristic searching of ant colony algorithm, the gateway selection was treated as an anycast service [18.] Analyzed different metaheuristic methods to consider the placement of mesh routers in WMNs. Taking connectivity and coverage problems, various schemes including genetic algorithm, simulated annealing, tabu search, and hill climbing were used to analyze their behaviors. The authors then used Friedman test to compare the simulation results. Here, it has been found that different algorithms work better than the others when subjected to different situations [19]. In their effort to maximize the serviced number of clients or subscribers in WMN, they developed load-based greedy algorithm integrated with load-based MCM algorithm to offer a delaycontrained and interference-free solution to construct multicast trees for the network [20]. Proposed a genetic algorithm to solve node placement problem in a wireless mesh network. By employing different mutation and selection operators as well as size of giant component, the authors considered Weibull distribution for the mesh clients [21]. Combined multi-objective Particle Swarm Optimization and Genetic Algorithm to devise a swarm-based algorithm to solve WMN planning problems of three models. The three multi-objective models 
are load-balanced, interference and flow-capacity models [22]. Adapted a new approach of placing mesh router nodes in WMN through heuristic tabu search. Acknowledging that a WMN optimization problem is oftentimes NP-complete, the authors explore the solution space at the same time avoid getting stuck in local minima by maximizing the size of giant components.

\section{PREVIOUS APPROACH}

Critical design factors of WMNs bring forth different challenging issues ranging from the physical layer to the application layer and it is apparent that many problems still remain [15]. Presented various heuristic methods to establish a wireless mesh network in IEEE 802.16j in an economical way. The work proposed a low cost RS and BS in order to compensate for bandwidth demand of the clients. Although the methods appear robust, they suffer from several limitations. After careful observations, it has been found that [15] failed to reflect the significance of distance between stations in the network. For example, if a node is dedicated to be a routing station or a base station to serve one subscriber, ignoring the node's distance from the subscriber might impact the quality of communication if the subscriber's distance from the node is small even if the cost is higher. Figure 2, is a graph showing possible links between nodes where the best solution is emboldened.

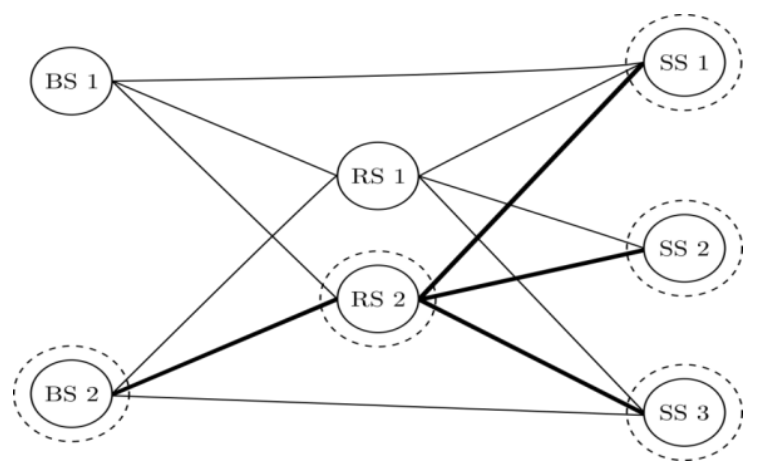

Figure 2. Example of an optimal solution. Adopted from [15]

It is known that digital signal attenuates during transmission. In instances where the distance between two nodes gets larger, the attenuation also grows bigger. In their effort to minimize the cost of establishing the network, they overlooked the affect that it does not always guarantee getting best performance inside the network. Such trade-off is common in optimization problems. This paper proposes an improvement for the method in network planning using heuristic algorithm where the primary purpose is to minimize the cost by taking into account the distance between nodes in WMN.

\section{PROPOSE APPROACH}

The problem at hand is a binary optimization problem. We have decided to approach the problem using Modified Binary Particle Swarm Optimization (MBPSO). MBPSO was chosen as it overcomes the unreasonable behavior of its predecessor, BPSO [23]. Suppose that the number of SSs is $n_{s}$, the number of RSs is $n_{r}$, and the number of BSs is $n_{b}$. The sum of components in each particle is equal to [15],

$$
\text { Sum of Components }=n_{r}+n_{b}+\left(n_{r} \times n_{s}\right)+\left(n_{b} \times n_{s}\right)+\left(n_{b} \times n_{r}\right)
$$

Whenever a new particle is generated, whether it is randomly generated (initialization phase or due to mutation) or due to the algorithm's progression, it must be checked for validity. If a station presented (by " 1 ") is invalid in real life due to it being out of the coverage area, it must be removed from the matrix that represents it (by turning it into " 0 ").

The aim of solving the problem is to minimize the cost of establishing a network. The fitness value of a network is as follows,

Fitness value $=\sum\left(\right.$ Cost of establishing $\left.\mathrm{RS}_{\mathrm{i}}\right)+\sum\left(\right.$ Cost of establishing $\left.\mathrm{BS}_{\mathrm{i}}\right)$ 
However, performance is not guaranteed just by minimizing the cost of establishing a network with regard to metrics such as PDR and E2E delay. [15] approached the problem from the perspective of cost but it ignored a crucial factor in network performance, i.e., the distance between network stations. Digital signals attenuate during transmission with respect to distance. The greater the distance, the greater the attenuation. Thus, ignoring such a consideration is detrimental to the performance of a network.

The mathematical model, "Model Based on Flow Conservation and Capacity" proposed by [15] is good and we have adapted it for the research at hand. Distance was taken into account during network planning by updating the objective function 2 as follows,

$$
\begin{aligned}
\text { Fitness Value }= & \sum\left(\text { Cost of establishing } \mathrm{RS}_{\mathrm{i}}\right)+\sum\left(\text { Cost of establishing } \mathrm{BS}_{\mathrm{i}}\right) \\
& +\sum(\text { Distances between each } \mathrm{BS} \text { and its served } \mathrm{RSs}) \\
& \left.+\sum \text { (Distances between each } \mathrm{BS} \text { and its served } \mathrm{SSs}\right) \\
& \left.+\sum \text { (Distances between each } \mathrm{RS} \text { and its served SSs }\right)
\end{aligned}
$$

Optimization will help minimize the previous function, which would balance minimizing cost and the total distance possible between network stations. Thus, improving the overall performance of the network.

\section{EXPERIMENTS AND RESULTS}

This section discusses the results from the conducted experiments in detail. A tripartite graph was used as the simulation model in MATLAB and is shown in Figure 3.

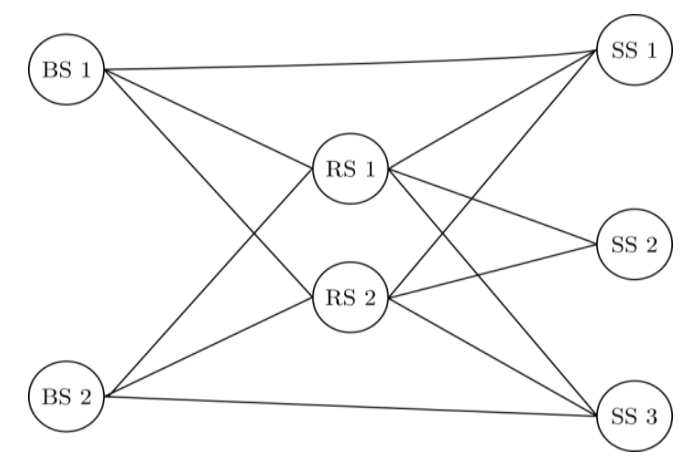

Figure 3. Tripartite graph representation of a sample problem. Adopted from [15]

Firstly, MBPSO had to be evaluated for which the MATLAB environment was chosen. The original objective function was used and the result presented as a $1 \times 20$ matrix,

Number of elements in result matrix $=2+2+2 \times 3+2 \times 3+2 \times 2=20$

The result had the following pattern: $\left(\mathrm{y}_{\mathrm{r}}, \mathrm{y}_{\mathrm{b}}, \mathrm{x}_{\mathrm{rs}}, \mathrm{x}_{\mathrm{bs}}, \mathrm{x}_{\mathrm{br}}\right)$, where elements:

1 and 2 represent $y_{r}$.

3 and 4 represent $y_{b}$.

5 to 10 represent $\mathrm{x}_{\mathrm{rs}}$.

11 to 16 represent $\mathrm{x}_{\mathrm{bs}}$.

17 to 20 represent $\mathrm{x}_{\mathrm{br}}$. See Table 1 .

Table 1. Particle Swarm Optimization parameters and chosen values

\begin{tabular}{lc}
\hline No. of Elements & 20 \\
\hline No. of Result Matrices & 50 \\
Velocity Range & {$\left[\begin{array}{ll}-4 & 4\end{array}\right]$} \\
No. of Iterations & 600 \\
$\mathrm{C} 1$ & 1 \\
$\mathrm{C} 2$ & 0.5 \\
$\mathrm{~W}_{\min }$ & 0.3 \\
$\mathrm{~W}_{\max }$ & 0.9 \\
Mutation Rate & 0.1 \\
\hline
\end{tabular}


The algorithm generated a solution with an establishment cost of 25 , as follows:

$\left[\begin{array}{llllllllllllllllllll}0 & 1 & 1 & 0 & 0 & 1 & 0 & 1 & 0 & 1 & 0 & 0 & 0 & 0 & 0 & 0 & 0 & 0 & 1 & 0\end{array}\right]$

where $\mathrm{y}_{\mathrm{r}}=\left[\begin{array}{l}0 \\ 1\end{array}\right], \mathrm{y}_{\mathrm{b}}=\left[\begin{array}{l}1 \\ 0\end{array}\right], \mathrm{x}_{\mathrm{rs}}=\left[\begin{array}{lll}0 & 0 & 0 \\ 1 & 1 & 1\end{array}\right], \mathrm{x}_{\mathrm{bs}}=\left[\begin{array}{lll}0 & 0 & 0 \\ 0 & 0 & 0\end{array}\right]$, and $\mathrm{x}_{\mathrm{br}}=\left[\begin{array}{ll}0 & 1 \\ 0 & 0\end{array}\right]$. In this solution, BS 1 and RS 2 form the backbone of the network, where BS 1 serves RS 2, which in turn serves SSs 1, 2, and 3. Upon updating the objective function, MBPSO was performed with the same parameters. In order to perform comparison between our approach and [15], the distances between the different network stations have to be known. These distances have been assumed and are presented in Tables (2-3). The distance between RS 1 and SS 2 (30) is less than that between RS 2 and SS 2 (80).

Table 2. Distances between BSs and RSs and SSs

\begin{tabular}{|c|c|c|c|c|c|}
\hline & $\mathrm{R}_{\mathrm{b}}$ & $\mathrm{R}_{\mathrm{b}}$ distance & $S_{b}$ & $\mathrm{~S}_{\mathrm{b}}$ distance & Coverage Zone Radius \\
\hline BS 1 & {$\left[\begin{array}{ll}1 & 2\end{array}\right]$} & {$\left[\begin{array}{ll}100 & 100\end{array}\right]$} & 1 & 75 & 150 \\
\hline BS 2 & {$\left[\begin{array}{ll}1 & 2\end{array}\right]$} & {$[100$} & 3 & 75 & 150 \\
\hline
\end{tabular}

Table 3. Distances between RSs and SSs

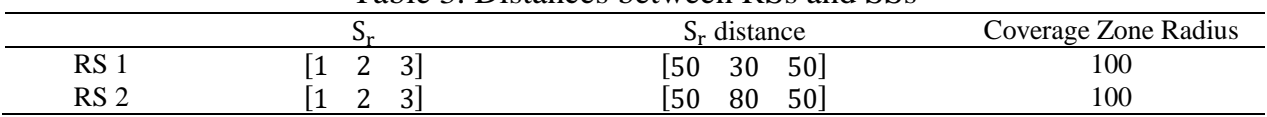

The experiment yields the following solution with an establishment cost of 35 ,

$$
\left[\begin{array}{llllllllllllllllllll}
1 & 0 & 1 & 0 & 1 & 0 & 1 & 0 & 1 & 0 & 0 & 0 & 0 & 0 & 0 & 0 & 1 & 0 & 0 & 0
\end{array}\right]
$$

where $\mathrm{y}_{\mathrm{r}}=\left[\begin{array}{l}1 \\ 0\end{array}\right], \mathrm{y}_{\mathrm{b}}=\left[\begin{array}{l}1 \\ 0\end{array}\right], \mathrm{x}_{\mathrm{rs}}=\left[\begin{array}{lll}1 & 1 & 1 \\ 0 & 0 & 0\end{array}\right], \mathrm{x}_{\mathrm{bs}}=\left[\begin{array}{lll}0 & 0 & 0 \\ 0 & 0 & 0\end{array}\right]$ and $\mathrm{x}_{\mathrm{br}}=\left[\begin{array}{ll}1 & 0 \\ 0 & 0\end{array}\right]$. The backbone of the network according to this solution is formed by BS 1 and RS 1, where RS 1 then serves SSs 1,2 , and 3 . The routes between stations for results obtained using the original and updated objective functions are presented in Tables (4-5).

Table 4. Routes using original objective function

\begin{tabular}{cc}
\hline Subscriber Station & Route \\
\hline SS 1 & RS 1-BS 1 \\
SS 2 & RS 1 - BS 1 \\
SS 3 & RS 1-BS 1 \\
\hline
\end{tabular}

Table 5. Routes using the updated objective function

\begin{tabular}{cc}
\hline Subscriber Station & Route \\
\hline SS 1 & RS 2-BS 1 \\
SS 2 & RS 2-BS 1 \\
SS 3 & RS 2-BS 1 \\
\hline
\end{tabular}

Secondly, real-world performance had to be evaluated using the PDR and E2E delay metrics. A simulation of a real network was carried out in MATLAB. Every SS would generate data packets to be sent to the allocated BS. The number of data packets and the interval of generation were determined using Poisson random variables. The number of data packets had a mean value of 6 whereas the interval of generation had a mean of $6 \mathrm{sec}$. We have assumed that each BS has a mechanism for detecting errors in received data packets. If errors exist, the BS will send a message to the source station so that it may re-send the affected data packets while the elapsed time is less than the packet life time $(0.5 \mathrm{sec})$. The channels between SS - RS, SS - BS, and RS - BS are based on the Gillbert-Elliot model. The quality of a channel is indirectly proportional to the distance between any two stations. Two experiments were carried out to compare the performance of the network with regard to PDR and E2E delay. The duration of each experiment was 1500 
sec of which $25 \mathrm{sec}$ was used for data logging. 60 samples for PDR and E2E delay values were logged and the results are discussed in the following section.

\section{EVALUATION AND DISCUSSION}

Figures (4-5) clearly show that taking distance into consideration has a positive impact on the performance of the network. The PDR values are better since RS 1 is used instead of RS 2; the distance between SS 2 and a routing station is less and thus signal quality with regard to SS 2 is improved. This also has a positive effect on the E2E delay; improved signal quality decreases the probability of errors in received signals which in turn decreases the number of acknowledgments and re-sending of data packets, thus reducing E2E delay time.

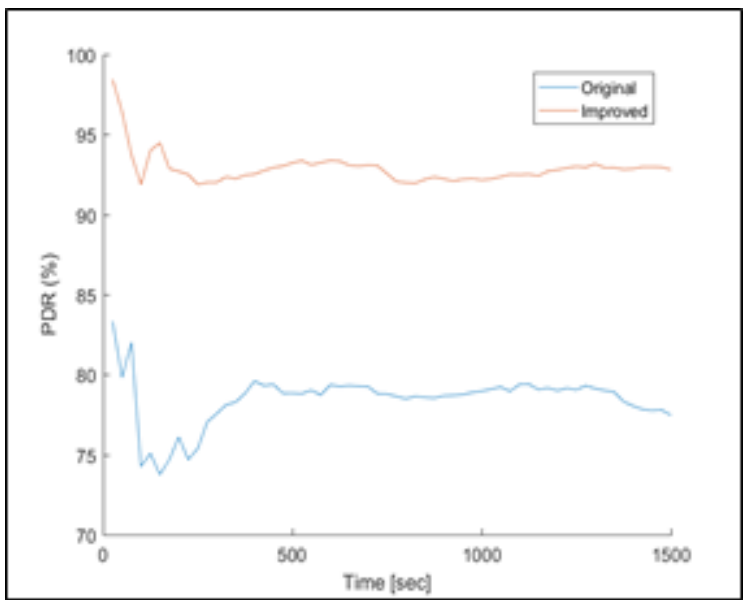

Figure 4. PDR comparison between original and modified objective function

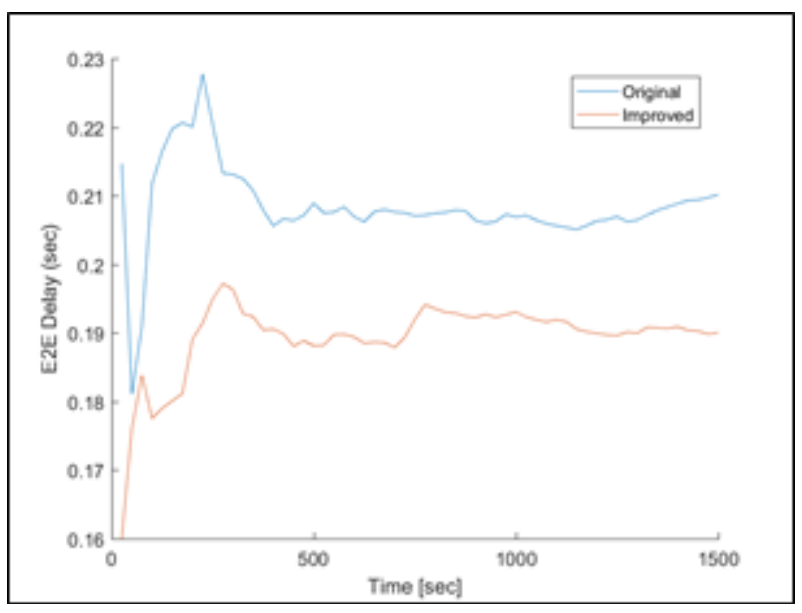

Figure 5. E2E Delay comparison between original and modified objective function

Finally, the finding of this study is based on the result as shown in Figures (4-5), this study has a positive effect on performance of the network through the quality of signal of routing station is less. Other hand, reducing the time delay through decreases the number of acknowledgments and re-sending of data packets.

\section{CONCLUSION}

Cost optimization is an area of research with regard to WMNs. This research was based upon improving the solution proposed by [15] in this regard. They have considered cost optimization without taking distance between nodes into consideration. This research considered the problem using a Modified Binary Particle Swarm Optimization (MBPSO) approach by updating the optimization function to take into consideration the distances between the different nodes. The results were positive and this approach showed noticeable improvement compared to the benchmark. The PDR showed an approximate increase of $17.83 \%$ whereas the E2E delay saw an approximate decrease of $8.33 \%$.

\section{ACKNOWLEDGEMENT} $\mathrm{UKM} / 02 / 3$

This work is supported by Ministry of Education of Malaysia, Grant no: FRGS/ 1/ 2015/ ICT04/

\section{REFERENCES}

[1] J. Coimbra, G. Schütz, and N. Correia. A game-based algorithm for fair bandwidth allocation in Fibre-Wireless access networks, Optical Switching and Networking, vol. 10, pp. 149-162, 2013.

[2] J. Coimbra, G. Schütz, and N. Correia. Energy efficient routing algorithm for fiber-wireless access networks: A network formation game approach, Computer Networks, vol. 60, pp. 201-216, 2014.

[3] F.L. Khaleel. Recruitment and Job Search Application, Universiti Utara Malaysia, 2011. 
[4] F.L. Khaleel, N.S. Ashaari, T.S. Meriam, T. Wook, and A. Ismail. The study of gamification application architecture for programming language course, in Proceedings of the 9th International Conference on Ubiquitous Information Management and Communication, 2015a, p. 17.

[5] F.L. Khaleel, N.S. Ashaari, T.S. Meriam, T. Wook, and A. Ismail. User-Enjoyable Learning Environment Based on Gamification Elements, in International Conference on Computer, Communication, and Control Technology (I4CT 2015), Kuching, Sarawak, Malaysia, 2015b, p. 221.

[6] F.L. Khaleel, N.S. Ashaari, T.S. Meriam, T. Wook, and A. Ismail. The Architecture of Dynamic Gamification Elements Based Learning Content, Journal of Convergence Information Technology, vol. 11, pp. 164-177, 2016a.

[7] F.L. Khaleel, T.S.M.T. Wook, N.S. Ashaari, and A. Ismail. Gamification Elements for Learning Applications, International Journal on Advanced Science, Engineering and Information Technology, in press, $2016 \mathrm{~b}$.

[8] G. Ma, R. Gong, Q. Li, and G. Yao. A Improved Particle Swarm Optimization Algorithm with Dynamic Acceleration Coefficients, Bulletin of Electrical Engineering and Informatics, vol. 5, 2016.

[9] S.M. Ali, P.S. Babu, and B. Gurusekhar. Reconfiguration with Simultaneous DG installation to Improve the Voltage Profile in Distribution Network using Harmony Search Algorithm, Bulletin of Electrical Engineering and Informatics, vol. 4, pp. 257-273, 2015.

[10] S. Middha and P. Singh. Channel Assignment with Greedy Algorithm for Wireless Mesh Network, Bulletin of Electrical Engineering and Informatics, vol. 5, 2016.

[11] X. Zhang and L. Huang. A Density Control Based Adaptive Hexahedral Mesh Generation Algorithm, Indonesian Journal of Electrical Engineering and Informatics (IJEEI), vol. 4, 2016.

[12] M. Anusha, S. Vemuru, and T. Gunasekhar. Transmission protocols in Cognitive Radio Mesh Networks, International Journal of Electrical and Computer Engineering, vol. 5, 2015.

[13] Y. Bejerano, K.T. Lee, S.J. Han, and A. Kumar. Single-path routing for life time maximization in multi-hop wireless networks, Wireless Networks, vol. 17, pp. 263-275, 2011.

[14] I.F. Akyildiz and X. Wang. A survey on wireless mesh networks, IEEE Communications magazine, vol. 43, pp. S23-S30, 2005.

[15] S.M. Khaled. Heuristic algorithms for wireless mesh network planning, Lethbridge, Alta.: University of Lethbridge, Dept. of Mathematics and Computer Science, c2012, 2012.

[16] R. Matam and S. Tripathy. Improved heuristics for multicast routing in wireless mesh networks, Wireless networks, vol. 19, pp. 1829-1837, 2013

[17] S. Ling, C. Jie, and Y. Xue-jun. Multi-path anycast routing based on ant colony optimization in multi-gateway WMN, in Computer Science and Education (ICCSE), 2010 5th International Conference on, 2010, pp. 1694-1698.

[18] T. Oda, Y. Liu, S. Sakamoto, D. Elmazi, L. Barolli, and F. Xhafa. Analysis of mesh router placement in wireless mesh networks using Friedman test considering different meta-heuristics, International Journal of Communication Networks and Distributed Systems, vol. 15, pp. 84-106, 2015.

[19] W.L. Yang, C.C. Kao, and C.H. Tung. Heuristic Algorithms for Constructing Interference-Free and DelayConstrained Multicast Trees for Wireless Mesh Networks, TIIS, vol. 5, pp. 269-286, 2011.

[20] A. Barolli, T. Oda, F. Xhafa, L. Barolli, P. Papajorgji, and M. Takizawa. WMN-GA System for Node Placement in WMNs: Performance Evaluation for Weibull Distribution of Mesh Clients, in Information Technology Convergence, ed: Springer, 2013, pp. 223-231.

[21] D. Benyamina, A. Hafid, N. Hallam, M. Gendreau, and J. Maureira. A hybrid nature-inspired optimizer for wireless mesh networks design, Computer Communications, vol. 35, pp. 1231-1246, 2012.

[22] F. Xhafa, A. Barolli, and M. Takizawa. A tabu search algorithm for efficient node placement in wireless mesh networks, in Intelligent Networking and Collaborative Systems (INCoS), 2011 Third International Conference on, 2011, pp. 53-59.

[23] J. Yang, H. Zhang, Y. Ling, C. Pan, and W. Sun. Task allocation for wireless sensor network using modified binary particle swarm optimization, IEEE Sensors Journal, vol. 14, pp. 882-892, 2014.

IJECE Vol. 7, No. 1, February $2017: 309-315$ 